\title{
Towards in-line detection of thermal polymer degradation
}

Polymers are an indispensable part of modern society, found in almost every product we use, including machine and engine components. But polymers degrade, and they need close this doesn't cause structural materials or devices to fail. We already have many ways to assess polymer degradation in the lab, but monitoring polymers while they're in use is much harder. In a new experiment, MSc Aler Wolfgang Binder and Martin Luther University HalleWittenberg along with ALTANA $A G$ and its division ELANTAS who initiated the research, have established the potential for a real-time sensor system to detect thermal degradation of a class of polymers called poly(ester imide)s (PEIs). Their new approach will be importan engines, where these materia engines, where these
are used for insulation.

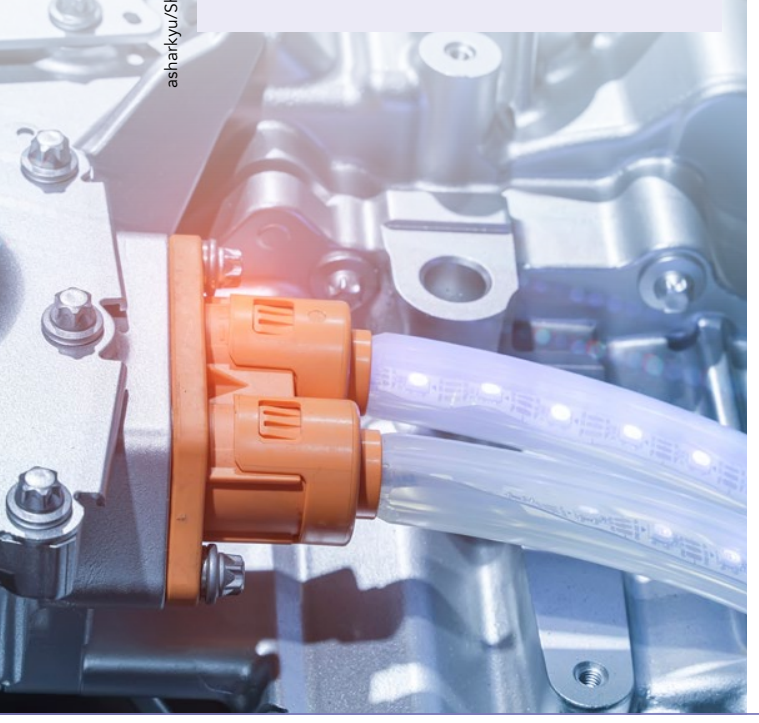

Dolymers - materials consisting of in almost every area of modern living. They are in our shopping bags, the clothes you're wearing reht now in in parts of your phone, your computer. and your car. The many useful properties of various polymers include being very strong and lightweight while being comparatively stiff, their resistance corrosion, lack of heat and electrical conductivity, and low cost. The easily adaptable properties of polymers such as tuning their reactivity, biocompatibility flexibility, and resistance, makes them suitable for a large number of applications around us. One such application is in the motors of electric cars.

POLYMERS AND ELECTRIC VEHICLES Sustainability is now a common goal, included in our ril sponse to the negat response, the use of electric vehicl is increasing, and these vehicles are confronting us with new challenges. Whit combustion engines have appropriate. sensors to indicate they need an oil topup or change, such sensors are still not available for electric cars, even though their components are also subject to we during operation.

Parts of electric motors are coated with special polymeric resins, which experience thermal degradation as the motor operates. As such coatings are required for insulation and mechanical immobilisation, their fallure could have of an consequences. The development indicate when the polymer has degrad to the point where it needs replacing would avoid unnecessary engine replacements and thus offer a valuabse

Professor Wolfgang Binder and MSc Alexander Funtan, from Martin Luther University Halle-Wittenberg, Germany

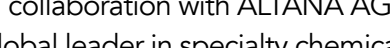
and its division ELANTAS, a leading manufacturer of insulating and protective materials with a focus on the global electrical and electronics industry, have designed a chemosensor for poly(ester imide)s (PEIs). PEls belong to a class of high-performance engineering thermosets which are used in a multitude of appliances such electrical tools, washing machines, explosion-proof motors, and driling machines. They are popules, so being abe to montical degradation of PEls is significant.

\section{CHALLENGES OF POLYMER} DEGRADATION MONITORING Polymers can be natural or synthetic, but mechanical force, or light, which naturally is a problem for the durability of our modern products. Because of this, polymers need constant monitoring to ensure the sustainability of our structural materials and devices. Depending upon the type of polymers, there are a variety of classical methods of ex-situ (off site) monitoring available, based on changes in molecular weights, mechanical properties, or changes in their insulating behaviour, foxample. How iver, examing the in operation, is much harder as many

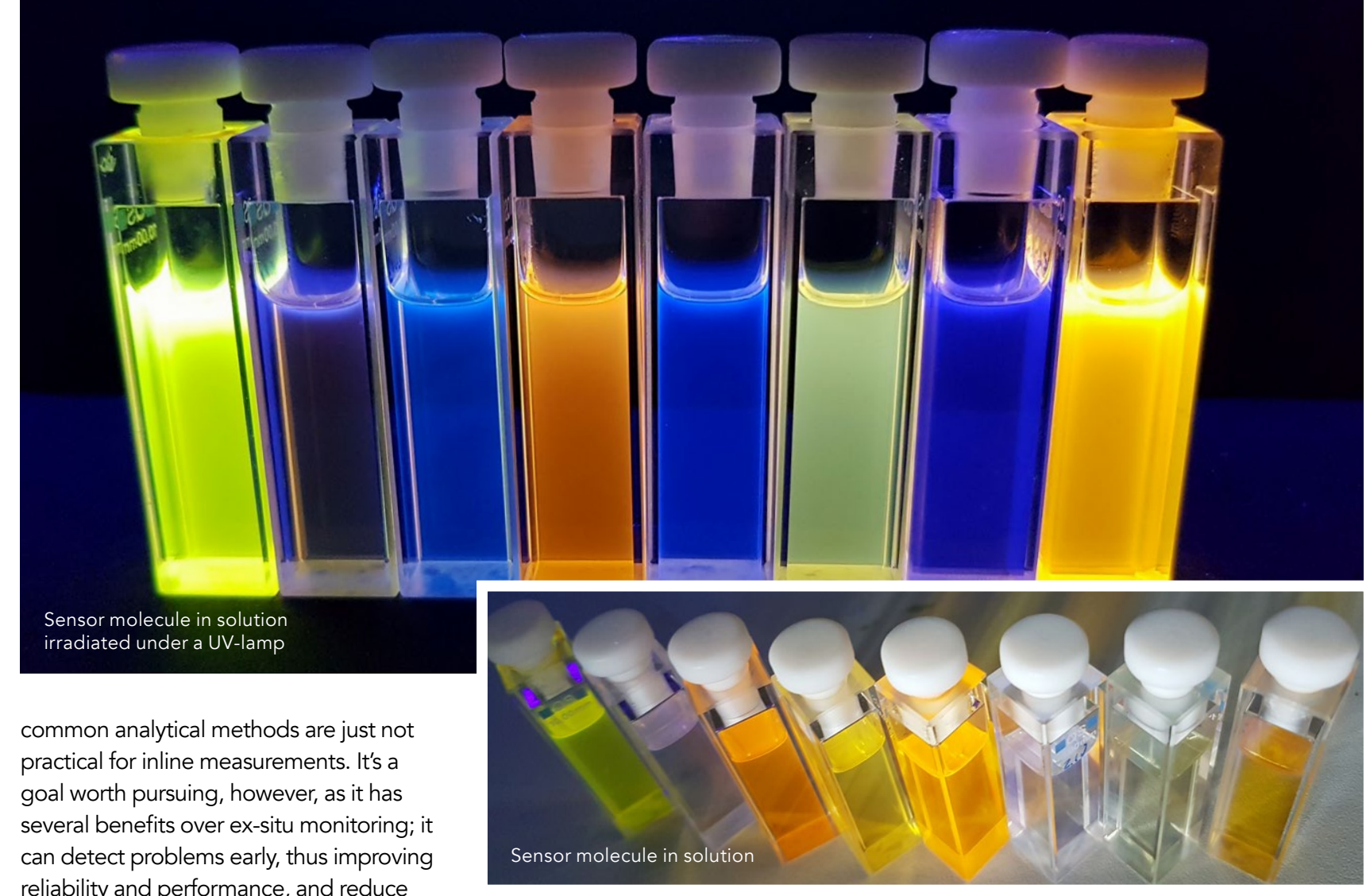

ms early, thus improving reliability and performance, and reduce

the risk of machine failure.

The development of an appropriate

Recently, sensor-based technology has been a focus of attention over are much easier to use in real-time investigations. Continuous detection of polymer degradation requires a trigger associated with the degradation process, so sensor technology is being develop based on the detection of chemical, ternal, mechanical, mass-sensitive or

\section{SENSING THERMAL}

Chemosensors in particular offer a

promising approach to the detection

of degradation products - molecules

continuously released during polymer decomposition. This approach requires with the degradation product to produc degradation product to detectable change; in this whose activation is accompanied by change of spectroscopic properties which can easily be observed using fuorescence spectroscopy

Binder and Funtan designed synthetic stilbene molecules to continuously sensor can avoid unnecessary engine replacements and offers a valuable contribution to sustainability

track the thermal degradation of a poly(esterimide)-polystyrene (PEI) resi PEls are known to hav high els a che high heat period of several years. The researchers chose to use the optical chemosensor, a trifluoroacetyl-functionalised stilbene dye (Stil-3, Stil-4) which, when embedded into the PEI matrix, reacts with the target molecule neopentyl ageing. This reaction is easily seen by a shift in wavelength, detected wonitoring the respoctoscopy. By avirgin ( he responses ofboth aving (unactivated) and activated were able to calculate the thermal degradation of the PEI.

TESTING IN SOLUTION AND SOLID STATE glycol (NPG) released during thermal

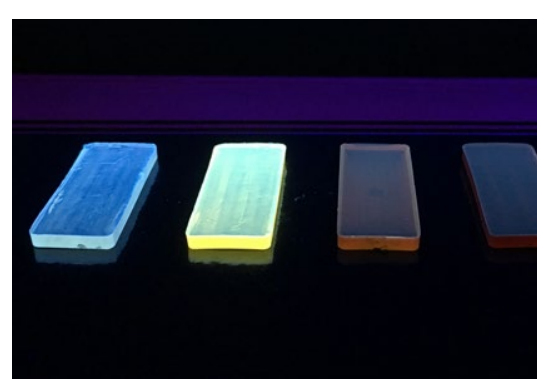
inder and Funtan first had to identify

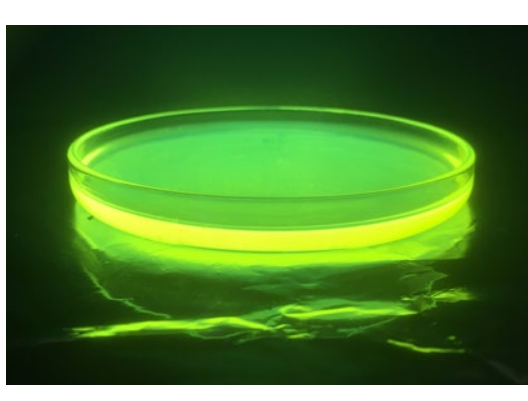
experience thermal degradation oy 


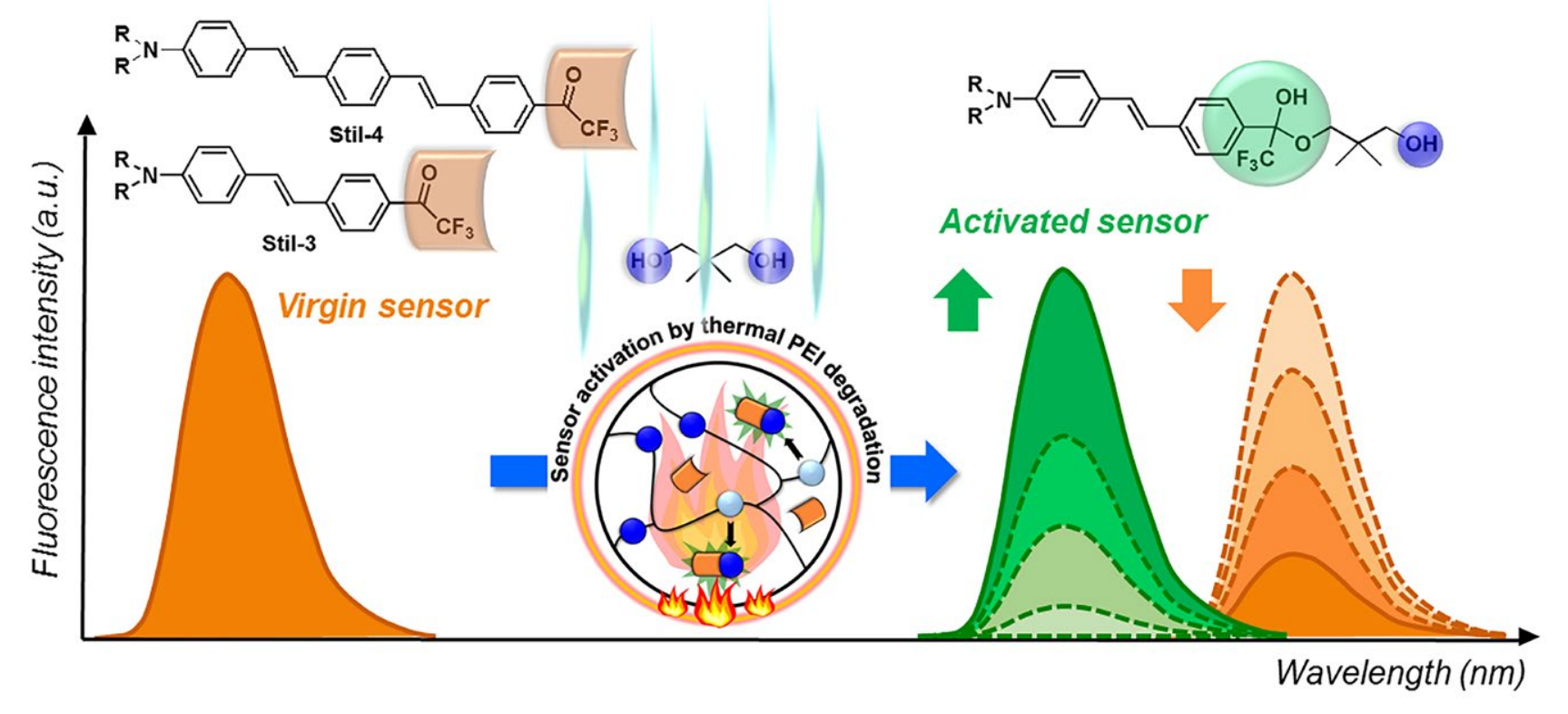

The activiation of the trifluoroacetyl functionalised sensor molecules Stil-3 and Stil-4, triggered by reaction with neopentyl glycol (NPG) released during
thermal degradation of the polylesterimide) PEE, can be detected by fluorescence spectroscopy $\left(R=-C_{6} H_{13}\right.$.

a suitable analyte released during the thermal decomposition
of the PEI. They conducted therm degradation experiments at 200 and $220^{\circ} \mathrm{C}$ which are representative temperatures for in-situ degradation. By
capturing the degradation products at capturing the degradation products at 500 to 5000 hof testing, anging from 500 to 5000 hours, and investigation

by nuclear magnetic resonance NPG as a possible target molecule To prove the suitability of the two stibere molecules, Stil-3 and Stil-4, they first conducted solution experiments, using different analytes at different concentrations, in particular amines and alcohols which are known to be given off during PEl thermal degradation; it is these degradation products that bind to the sensor molecules and produce an observable change in their fluorescence spectra. Both Stil-3 and Stil-4 were seen to be activated by n-butylamine and by alcohols, responding with a shift of the emission maximum in just seconds The researchers subsequenty focused on NPG as the main analyte for thermal degradation, which produced a strong
The release of neopentyl glycol, asociated with thermal degradation of PEl-resins, triggers sensor

\section{vation subsequently monitored by}

fluorescence spectroscopy

Having first proven sensor response and detection of the chosen detection system
in the solid state on a model system, a in the solid state on a model system, a poly(styrene divinybenzene) resin (PS/ poly(styrene divinybenzene) resin (PS/
DVB), Binder and Funtan turned their attention to solid PEl resin. They used two formulations of PEI provided by ALTANA $A G$ and added either $S$ til-3 or Stil-4. A with their PS/DVB test, they stored the specimens in $n$-butylamine solutions and used fluorescence spectroscopy to detect sensor activation. The responses of both sensor molecules within PEl confirmed their findings from the preliminary experiments in solution.

NEOPENTYL GLYCOL AND SOLID PEI RESPONSE Finally, Binder and Funtan examined sensor activation in solid PEl under' real life' conditions. Both Stil-3 and Stil 4 were embedded in PEl samples and exposed to NPG to determine how the sensor molecules would react. The samples exposed to temperature or in gas phase at $180^{\circ} \mathrm{C}$ Control samples of PEl without sensor molecules were given the same treatment. They found that the sample at room

emperature produced a decrease in luorescence of $8 \%$ compared to a decrease of $26 \%$ in the heated samp control experiment.

\section{LFECYCLE ENHANCEMENT} AND WASTE REDUCTION By successfully embedding these senso Binder and Funtan have demonstrated that this approach works well under different conditions, in both solution and solid state. This offers a potentially powerful tool for tracking the longterm degradation of PEl-impregnated protective coatings in real time; an important step towards prolonging the life of electric motors and enhancing the sustainability of all electric vehicles. The next step in this work is to investigate the in-situ activation of engine-working conditions.

\section{Behind the Research}

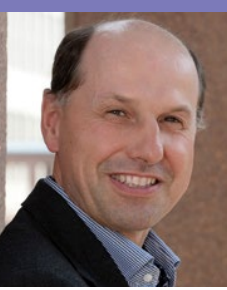

Professor

Wolfgang

Binder

MSc Alexander Funtan

E: wolfgang.binder@chemie.uni-halle.de T: +49 (0)345/55 25930 E: alexander.funtan@chemie.uni-halle.de T: +49 (0)345/55 25916 W: www.chemie.uni-halle.de/bereiche_der_chemie/technische_chemie/33918_1050877 W: www.macrochem.uni-halle.de

\section{Research Objectives}

The synthesis and development of novel polymers with applications in the areas of self-healing materials, life-cycle enhancement biomedicine, charge-storage materials and novel concepts of circular economy.

\section{Detail}

Address

Institute of Chemistry, Division Danckelmann-Platz 4

-06120 Halle

Bio

Wolfgang $H$ Binder became a full Prefessor of macromolecular chemistry Wittentin Luther University HalleWittenberg in 2007. After a PhD in Organic chemistry, and post-doctoral and the University of Vienna, Austria,
he became associate professor at Vienna University of Technology, TUWien, in 2004

Alexander Funtan gained an MSC investigating poly(ionic) liquids for in Professor Binder's Macromolecular Chemistry group, at Martin Luther University Halle-Wittenberg in 2017 polymer degradation.

\section{References}

Funtan A., Michael P., Rost S., Omeis J., Lienert K. and Binder W. (2021) Self-Diagnostic Polymers - Inli Poly(ester imide)s. Adv Mater, 33 (18) 2100068 (9)

Personal Response

What further work will be needed to bring these

II There is intense interest from manufacturers, especially in the automotive and engine industries, technology. Upscaling and long-term testing are in progress to validate data from laboratory expere in and move into technology, including processdevelopment and long-term stabilities. ALTANA AG, as the PEI manufacturer and patent owner, will start such
collaborations to bring the product to market.

MARTIN-LUTHER

UNIVERSITÄT

HALLE-WITTENBERG

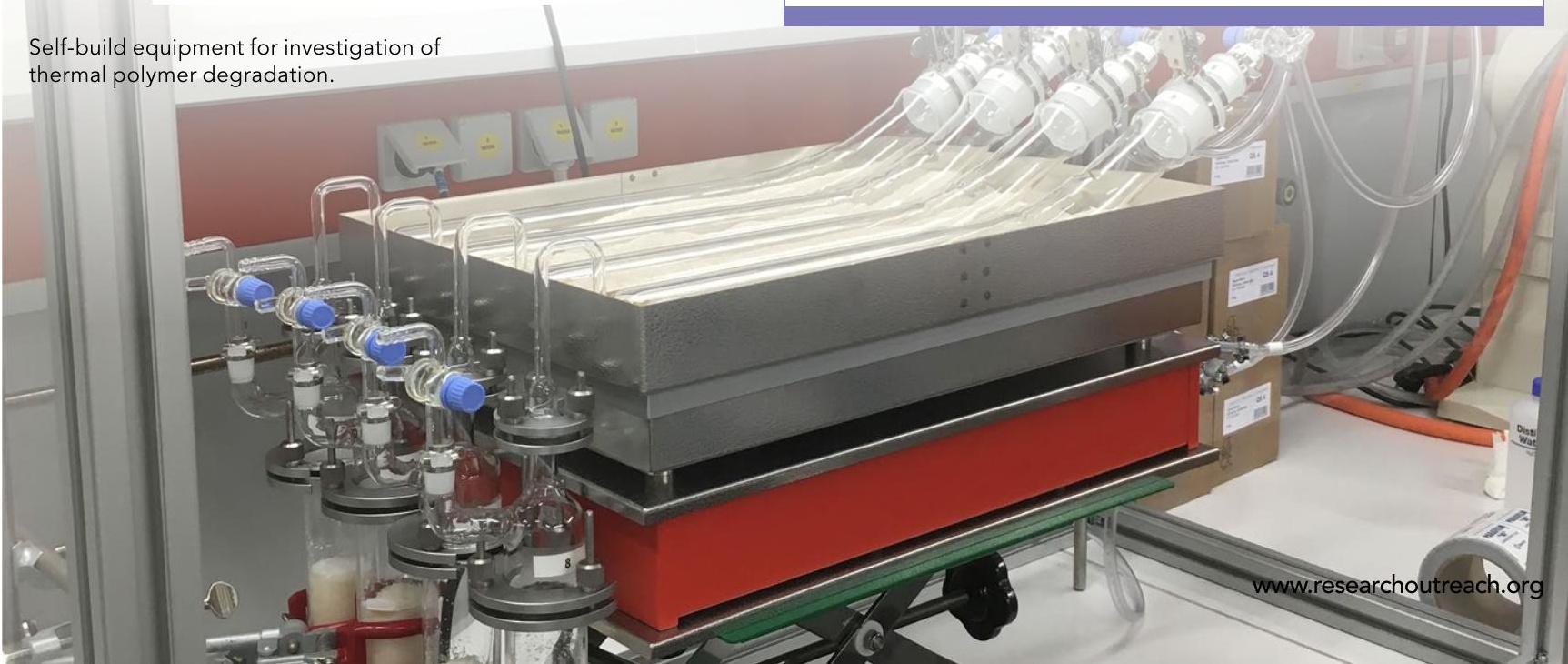

